\title{
Promoting Dolichoderus thoracicus as an Agent to Disperse Trichoderma sp., a Fungus that Controls the Black Pod Disease, Central Sulawesi - Indonesia
}

\author{
Tjandra Anggraeni ${ }^{1}$, Umrah $^{2}$, Rizkita R. Esyanti ${ }^{1}$ \& I Nyoman P. Aryantha ${ }^{1}$ \\ ${ }^{1}$ Physiology, Developmental Biology \& Biomedics Research Group, \\ School of Life Sciences and Technology, Institut Teknologi Bandung, \\ Jalan Ganesa 10 Bandung 40132, Indonesia \\ ${ }^{2}$ Faculty of Mathematic and Natural Sciences, Universitas Tadulako \\ Jalan Soekarno Hatta km. 9, Palu, Sulawesi Tengah 94118, Indonesia \\ Email: tjandra@sith.itb.ac.id
}

\begin{abstract}
In this study, we propose to use Dolichoderus thoracicus to act as a double agent; not only as an agent to control cocoa plant pests, but also as an agent for distributing Trichoderma sp., a fungus that suppresses the development of the black pod disease caused by Phytophthora palmivora. In the experiments, D. thoracicus was more attracted to honey-soil media (M5) and coconut waste pulp-soil media (M6). However, 10\% sucrose-potato-soil media (M3) was the best media for growing Trichoderma sp. Morphological study showed that spores attached to many parts of $D$. thoracicus. The efficiency of $D$. thoracicus in distributing the spores compared to the conventional method of using a sprayer was measured. The results showed that the growth of $P$. palmivora was suppressed by $83.33 \%$, which is not significantly different from when Trichoderma was dispersed through spraying (87\% suppressed). It was concluded that $D$. thoracicus can act as a double agent and can be used to disperse Trichoderma sp.
\end{abstract}

Keywords: Black pod disease; cocoa plantation; D. thoracicus; P. palmivora; Sulawesi; Trichoderma sp.

\section{$1 \quad$ Introduction}

Just like the invasion of pests such as $C$. cramerella and $H$. theobroma, the black pod disease is a major problem for cacao plantations in Central Sulawesi, Indonesia. Altogether they cause significant losses in production of up to $90 \%$. The black pod disease is caused by the fungus $P$. palmivora, which can be dispersed quickly by several media, including wind and water, although the cocoa black ant, $D$. thoracicus, is known to be the main contributor in its dispersal. The presence of $D$. thoracicus, however, is important since these ants can control both $C$. cramerella and $H$. theobroma [1]. In our previous study [2], it was shown that one local species of Trichoderma sp., based on their performance in sporulation, antagonistic ability, was effective in suppressing the development of P. palmivora. This finding has already been applied in the

Received September $24^{\text {th }}, 2013,1^{\text {st }}$ Revision October $31^{\text {st }}, 2013,2^{\text {nd }}$ Revision November $26^{\text {th }}, 2013,3^{\text {rd }}$

Revision March $7^{\text {th }}, 2014$, Accepted for publication March $7^{\text {th }}, 2014$.

Copyright () 2014 Published by ITB Journal Publisher, ISSN: 2337-5760, DOI: 10.5614/j.math.fund.sci.2014.46.1.4 
field; Trichoderma sp. spores have been applied to suppress the growth of $P$. palmivora on the cocoa pod using sprayers. Inspired by the work of Sutton \& Peng [3], who succeeded in using Botrytis cinerea as an agent for biological control in a strawberry plantation, we attempted to use $D$. thoracicus as an agent to disperse Trichoderma sp. spores. The aims of this research were to find the best soil composition to grow Trichoderma sp. as well as to attract $D$. thoracicus, but also to evaluate the ability of $D$. thoracicus to disperse the Trichoderma sp. spores, as well as their effectiveness in suppressing the development of $P$. palmivora.

\section{$2 \quad$ Methods}

\subsection{D. thoracicus and Trichoderma sp.}

The D. thoracicus were collected from the field using mock nests (a piece of bamboo of approximately $5 \mathrm{~cm}$ in diameter and $40 \mathrm{~cm}$ in length, filled with coconut leaf and spread with honey), which were put on a branch of a cocoa plant for about 4 weeks to grow a colony. The colony of $D$. thoracicus was then reared in the laboratory. Before use, the ants were put into a honey contained sterile jar to adapt for $24 \mathrm{~h}$. The Trichoderma sp. used was from a collection of the Plant Pest and Disease Laboratory in Tadulako University, Palu, Indonesia. It was prepared in solid tablet form with $10.95 \times 10^{20}$ colony-forming units (cfu), according to the method from Umrah, et al. [2].

\subsection{Media for Trichoderma sp. Growing}

Samples of thirty grams of sterile soil each were mixed with $10 \mathrm{~mL}$ of six different sterile media according to Table 1 . Except for M0, all media were mixed with $10.95 \times 10^{20} \mathrm{cfu}$ of Trichoderma sp. Before use, these mixtures were then incubated at room temperature for $48 \mathrm{~h}$.

Table 1 Variation of media for growing Trichoderma sp. before dispersion by D. thoracicus or sprayer.

\begin{tabular}{cccc}
\hline Code & Variation of media & $\begin{array}{c}\mathbf{1 0 0} \text { mL media : with / } \\
\text { without 10,95 X 10 } \\
\text { of } \mathbf{~ T r i c h o d e r m a ~} \mathbf{~ s p . ~}\end{array}$ & Dispersed by \\
\hline M0 & Water & Without & D. thoracicus \\
M1 & Water & With & Sprayer \\
M2 & Water & With & D. thoracicus \\
M3 & $10 \%$ sucrose - potato & With & D. thoracicus \\
M4 & $10 \%$ sucrose & With & D. thoracicus \\
M5 & $10 \%$ honey & With & D. thoracicus \\
M6 & $10 \%$ coconut waste pulp & With & D. thoracicus \\
\hline
\end{tabular}




\subsection{Simulated Track and Preference of D. thoracicus for Variant of Media}

A simulated track of $D$. thoracicus's journey from soil to cocoa pod with a mock nest was prepared, as shown in Figure 1. In order to keep the ants on the track, each track was put inside a $70 \mathrm{~cm}$ x $40 \mathrm{~cm}$ x $40 \mathrm{~cm}$ container filled with about $1 \mathrm{~cm}$ height of water.

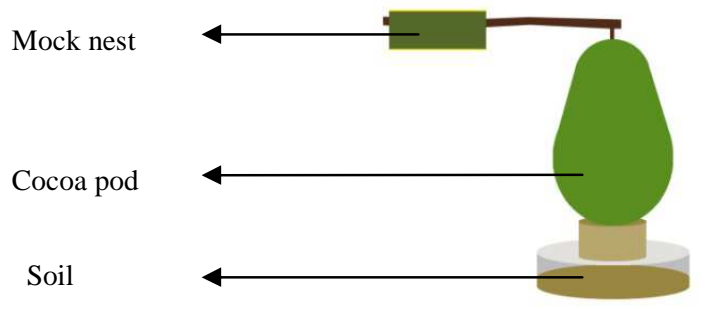

Figure 1 Simulated track $-D$. thoracicus route from soil to cocoa pod with mock nest.

Five different media (M2, M3, M4, M5, M6) were tested for the preference of D. thoracicus (Table 1). On each track, 600 D. thoracicus individuals were infested and the percentage of the ant activity in the media tested was recorded. $0.1 \mathrm{~g}$ of test medium was diluted with water and then cultured on Potato Dextrose Agar for 48 hours. The number of Trichoderma sp. in the medium was then counted according to the plating method by Cappuccino \& Sherman [4].

\subsection{Position and Number of Trichoderma sp. attached to $D$. thoracicus}

Two hundred individuals of $D$. thoracicus on the track containing the medium to be tested were infested for $2 \times 24$ hours. Samples of D. thoracicus were then examined under a microscope to identify the position of the attached spores. All the ants were then washed with $100 \mathrm{~mL}$ of sterile water to release the spores from their body. The spores in the water were cultured in Potato Dextrose Agar for 48 hours and the number of Trichoderma sp. was then counted according to the plating method by Cappuccino \& Sherman [4].

\subsection{Effectiveness of $\boldsymbol{D}$. thoracicus in Distributing Trichoderma sp. and Suppressing $\boldsymbol{P}$. palmivora}

The cocoa pods from the different treatments were each washed with $100 \mathrm{~mL}$ of sterile water. The spores in the water were cultured in Potato Dextrose Agar for 48 hours and the number of Trichoderma sp. in the water was then counted according to the plating method by Cappuccino \& Sherman [4]. The cocoa 
pods, which were previously contaminated with Trichoderma sp. according to the treatments in Table 1, were then inoculated with P. palmivora and incubated for 24 hours. The effectiveness of disease suppression was calculated by measuring the area of the black pod disease.

\section{$3 \quad$ Result and Discussion}

\subsection{Preference of $\boldsymbol{D}$. thoracicus to Variant of Media}

As shown in Figure 2, D. thoracicus was able to come on all soil media tested. However, honey-soil media (M5) attracted more insects than any of the other media tested. Statistical analysis showed that there was a significant difference with the other media tested $(\mathrm{p}<0.05)$ except for the combination of soil with $10 \%$ coconut waste pulp (M6), which also attracted D. thoracicus but not significantly differently from M3 or M5. Volatile chemicals from the soil media were probably the main factor in attracting $D$. thoracicus. The preference itself is influenced by many factors, including the need for energy, growth, and development [5].

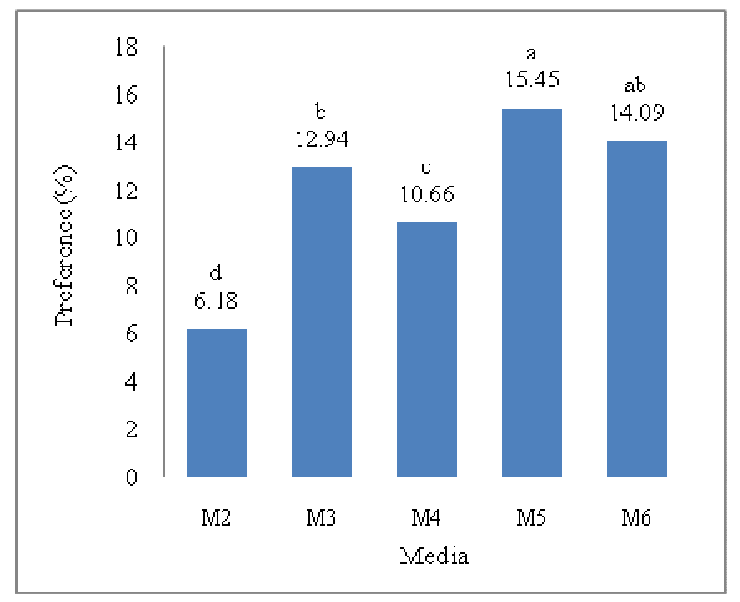

Figure 2 Preference of $D$. thoracicus to variant of media. M2: water, M3: 10\% sucrose-potato-soil, M4: 10\% sucrose-soil, M5: 10\% honey-soil, M6: $10 \%$ coconut waste pulp-soil.

\subsection{Number of Trichoderma sp. in the Media}

$10 \%$ sucrose-potato-soil medium (M3) was the best medium for growing Trichoderma sp., as shown in Figure 3. The number attained was $97.29 \mathrm{cfu}$ and statistical analysis showed that there was a significant difference with the other 
media tested $(\mathrm{p}<0.05)$. The results also indicated that sucrose-potato is a readyto-use element for growing Trichoderma sp.

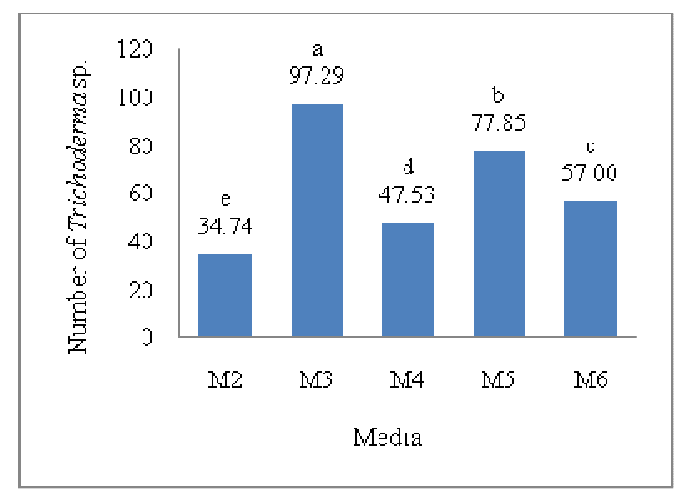

Figure 3 Number of Trichoderma sp. (cfu) in the variants of media. M2: water, M3: $10 \%$ sucrose-potato-soil, M4: 10\% sucrose-soil, M5: 10\% honeysoil, M6: $10 \%$ coconut waste pulp-soil.

\subsection{Position and Number of Trichoderma sp. Attached to $D$. thoracicus}

During the experiment, $D$. thoracicus traveled all the time from the mock nest to the soil through the cocoa pod. This activity caused the Trichoderma sp. spore in the soil to attach to the D. thoracicus body, including legs, antennas, head, thorax and abdomen (Figure 4). As the insects were always traveling, the spores were taken accidently to other places, including the cocoa pod.

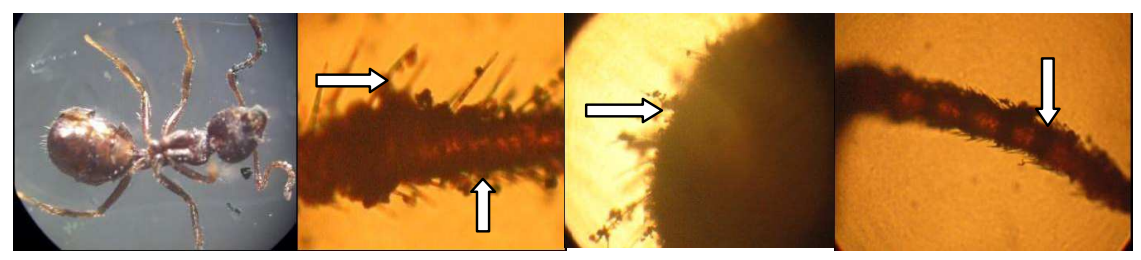

Figure 4 Position of Trichoderma sp. attached to D. thoracicus (arrows).

The number of Trichoderma sp. spores attached to the D. thoracicus body varied among the media tested. 10\% sucrose-potato-soil media (M3) gave the highest number of spores attached to D. thoracicus with $87.43 \mathrm{cfu}$ (Figure 5). This number was significantly different from the other media tested $(\mathrm{p}<0.05)$. The number of spores attached was related to the number of Trichoderma sp. spores in the media, the highest of which which came from $10 \%$ sucrose-potatosoil media (M3). 


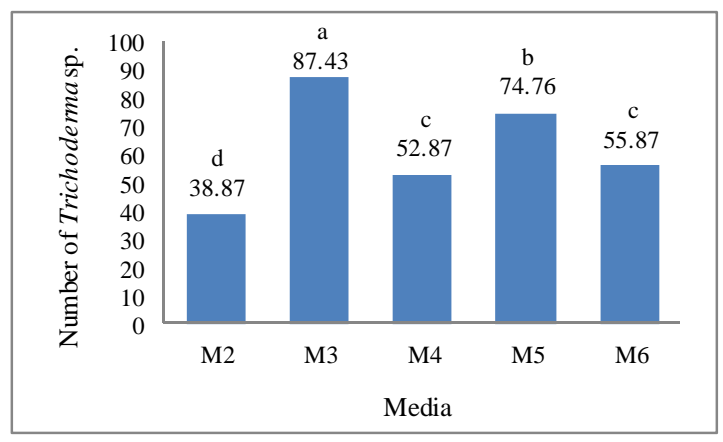

Figure 5 Number of Trichoderma sp. (cfu) attached to D. thoracicus for variant of media. M2: water, M3: 10\% sucrose-potato-soil, M4: 10\% sucrosesoil, M5: 10\% honey-soil, M6: 10\% coconut waste pulp-soil.

\subsection{Number of Trichoderma sp. in Cocoa Pod}

During travelling from mock nest to media, spores attached to the D. thoracicus body fell off and were deposited on the cocoa pod. The number of Trichoderma sp. spores on the cocoa pod using a spraying method (M1), which was 18.00 cfu, was not significantly different from the number of Trichoderma sp. spores on the cocoa pod that were deposited by $D$. thoracicus from the $10 \%$ sucrosepotato-soil media (M3), which was $15.77 \mathrm{cfu}$. However, this number was significantly different from that of the other media, as shown in Figure 6.

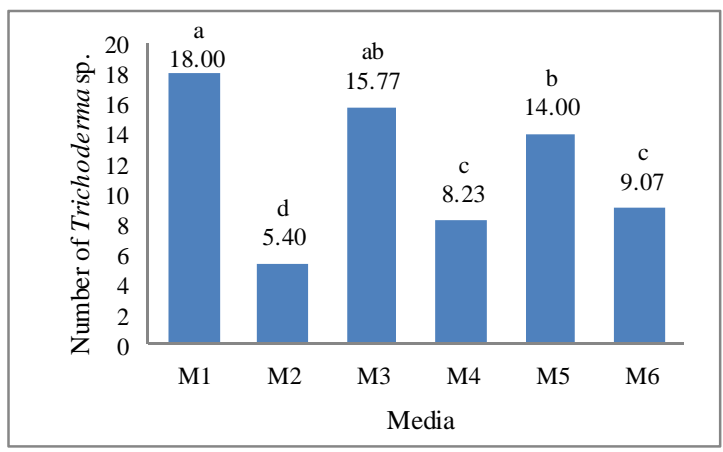

Figure 6 Number of Trichoderma sp. (cfu) from variant of media deposited in cocoa pod. M1: water with Trichoderma sp. dispersed by sprayer, M2: water with Trichoderma sp. dispersed by D. thoracicus, M3: 10\% sucrosepotato-soil with Trichoderma sp. dispersed by D. thoracicus, M4: 10\% sucrose-soil with Trichoderma sp. dispersed by D. thoracicus, M5: 10\% honey-soil with Trichoderma sp. dispersed by D. thoracicus, M6: $10 \%$ coconut waste pulp-soil with Trichoderma sp. dispersed by $D$. thoracicus. 


\subsection{Effectiveness of $D$. thoracicus in Distributing Trichoderma sp. and Suppressing $P$. palmivora}

Since the number of Trichoderma sp. spores on the cocoa pods was not significantly different between experiment M1 and M3, similar results were also achieved by Trichoderma sp. in suppressing the growth of $P$. palmivora. Spraying Trichoderma sp. spores (M1) gave $87.01 \%$ in $P$. palmivora growth suppression, which was not significantly different from when Trichoderma sp. spores were taken by $D$. thoracicus from $10 \%$ sucrose-potato-soil media (M3), which gave $85.18 \%$ suppression. As a result, the spread of the black pod disease on the cocoa pod was reduced as well, as shown in Figure 7. There was no significant difference between M1 and M3 whether Trichoderma sp. spores were dispersed by sprayer or by D. thoracicus from $10 \%$ sucrose-potato-soil media, respectively (Figure 8).
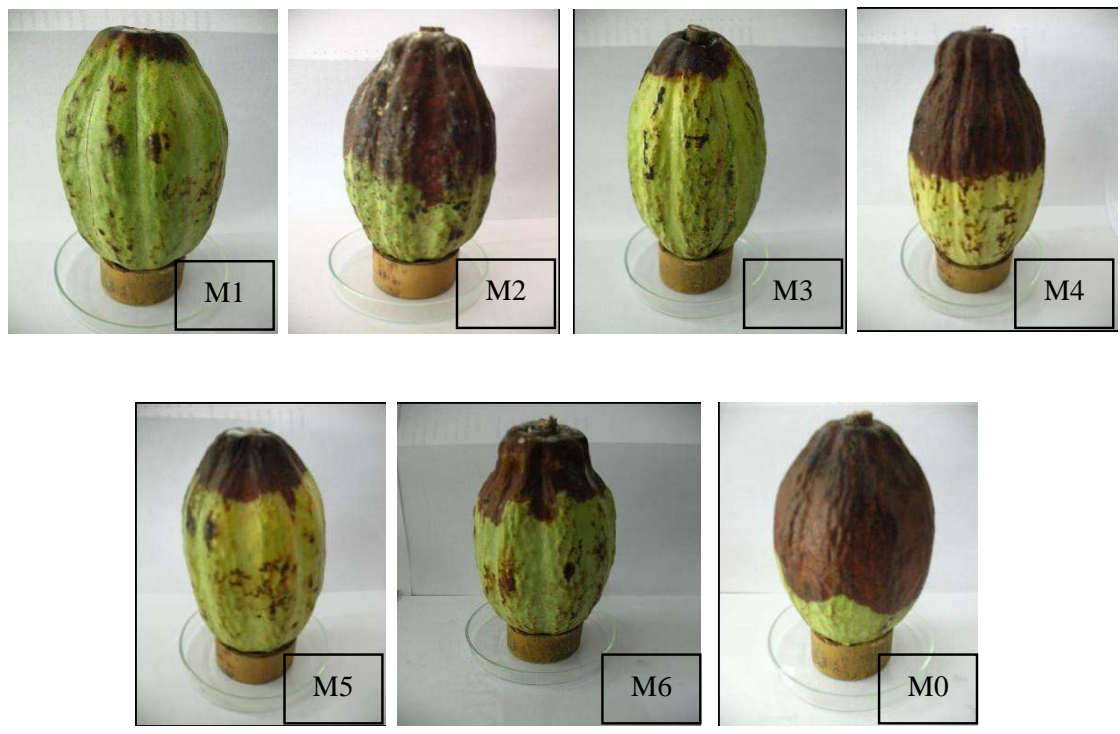

Figure 7 Growth of the black pod disease in cocoa pods. M1: water with Trichoderma sp. dispersed by sprayer, M2: water with Trichoderma sp. dispersed by $D$. thoracicus, M3: 10\% sucrose-potato-soil with Trichoderma sp. dispersed by $D$. thoracicus, M4: 10\% sucrose-soil with Trichoderma sp. dispersed by $D$. thoracicus, M5: 10\% honey-soil with Trichoderma sp. dispersed by $D$. thoracicus, M6: $10 \%$ coconut waste pulp-soil with Trichoderma sp. dispersed by $D$. thoracicus, M0: water without Trichoderma sp. dispersed by $D$. thoracicus. 


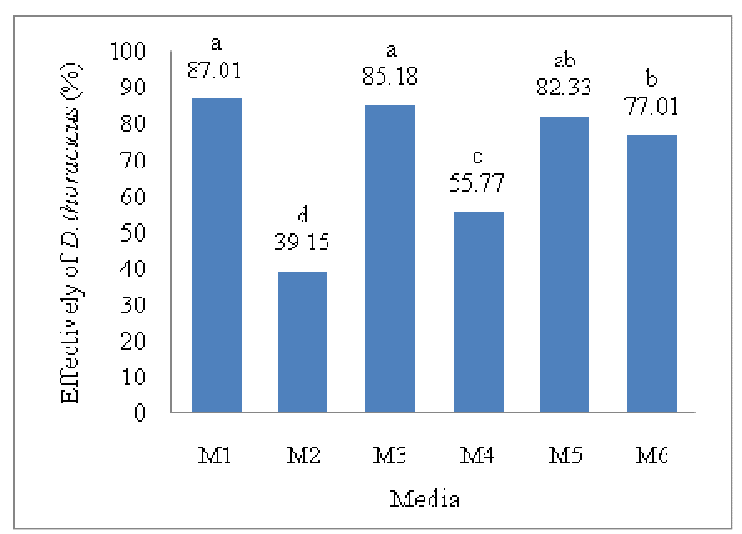

Figure 8 Effectiveness of $D$. thoracicus in distributing Trichoderma sp. and suppressing the growth of $P$. palmivora. M1: water with Trichoderma sp. dispersed by sprayer, M2: water with Trichoderma sp. disperse by $D$. thoracicus, M3: 10\% sucrose-potato-soil with Trichoderma sp. disperse by D. thoracicus, M4: 10\% sucrose-soil with Trichoderma sp. disperse by $D$. thoracicus, M5: 10\% honey-soil with Trichoderma sp. dispersed by $D$. thoracicus, M6: 10\% coconut waste pulp-soil with Trichoderma sp. dispersed by D.thoracicus.

\section{Conclusion}

$10 \%$ sucrose-potato-soil media (M3) was the best media for growing Trichoderma sp. Trichoderma sp. spores attached to the D. thoracicus body and were dropped onto the cocoa pod, therefore D. thoracicus can be used as an agent to disperse Trichoderma sp. There was no significant difference in effectiveness with the more commonly used spraying methods.

\section{Acknowledgments}

Many thanks to STORMA (Stability of Rainforest Margins), University of Tadulako for the research funding, and also to the School of Life Sciences and Technology ITB for all research facilities.

\section{References}

[1] Ashary, A. \& Pasaru, F., Mass Rearing Technique and Predator Application of Dolichoderus thoracicus for Cacao Pod Borer (Conopomorpha cramerella) Controling in Small Holder Plantations, J. Agroland, 15(4), pp. 278-287, 2008. 
[2] Umrah, Anggraeni, T., Esyanti, R.R. \& Aryantha, I.P., Development of Trichoderma sp. Inoculated Formulae as a Biological Agent to Control Black Pod Disease (Phytophthora palmivora, E.J. Butler) (Text in Indonesian), J. Agrisains, 10(2), pp. 72-82, 2009.

[3] Sutton, J.C. \& Peng, G., Manipulation and Vectoring of Biocontrol Organisms to Manage Foliage and Fruit Diseases in Cropping Systems, Annu. Rev. Phytopathol., 31, pp. 473-493, 1993.

[4] Cappuccino, J.G. \& Sherman, N., Microbiology a Laboratory Manual, Cummings Publ. Co. Inc., pp. 1-124, 1987.

[5] Ho, C.T. \& Khoo, K.C., Partners in Biological Control of Cocoa Pests; Mutualisms between Dolichoderus thoracicus (Hymenoptera: Formicidae) and Cataenococus hispidus (Hemiptera: Pseudococcidae), Bull. Entomol. Res., 87, pp. 461-470, 1997. 